\title{
Correction to: Sant'Imbenia (Alghero): further archaeometric evidence for an Iron Age market square
}

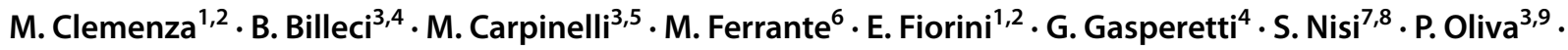 \\ V. Sipala ${ }^{3,5} \cdot$ P. R. Trincherini ${ }^{8} \cdot$ I. M. Villa ${ }^{1,10,11} \cdot$ M. Rendeli $^{3,5}$ (D)
}

Published online: 13 November 2021

(C) The Author(s) 2021

\section{Correction to: Archaeological and Anthropological Sciences https://doi.org/10.1007/s12520-021-01425-x}

The original online version of this article was revised:

Data in Tables 9 and 12 relating to the Catalan Coastal Ranges mines come from:

Montero Ruiz I, (2017) La Solana del Bepo from an archaeometallurgical perspective. Rafel N, Soriano I, Delgado-Raack $\mathrm{S}$ (edd) A prehistoric copper mine in the NothEast of the Iberian Peninsula: Solana del Bepo (Uldemolins, Tarragona). Revista de Arqueologia de Ponet, extra 2: 65-79;

instead of:
Antolinos Marin JA, Domergue C, Manteca JI, Palero Fernández FJ, Quarati P, Rico C, Stefanile M, Trincherini PR (in press) Lateres Plumbei Hispani. Production et commerce du plomb hispanique à l'époque romaine (iie s. av. J.-C. - iie s. ap. J.-C.), Ed. Casa de Velásquez, Madrid. (accepted November 2020)".

The original article has been corrected.

Publisher's note Springer Nature remains neutral with regard to jurisdictional claims in published maps and institutional affiliations.

The original article can be found online at https://doi.org/10.1007/ s12520-021-01425-x.

\section{Rendeli}

rendeli@uniss.it

1 Dipartimento di Fisica G. Occhialini, Università degli Studi Di Milano Bicocca, Piazza della Scienza 3, 20126 Milano, Italy

2 Istituto Nazionale di Fisica Nucleare-Sez. Milano Bicocca, Piazza della Scienza 3, 20126 Milano, Italy

3 Università degli Studi di Sassari, Piazzà Universita 21, 07100 Sassari, Italy

4 Ministero della Cultura-Soprintendenza Archeologia, Belle Arti e Paesaggio per le Province di Sassari e Nuoro, Piazza Sant'Agostino 2, 07100 Sassari, Italy

5 Laboratori Nazionali del Sud-Istituto Nazionale di Fisica Nucleare, Via Santa Sofia 62, 95123 Catania, Italy

6 Trace Technologies, Via I. Silone 6, 64015 Nereto, TE, Italy
7 Servizio di Chimica, Laboratorio Nazionale del Gran Sasso-Istituto Nazionale di Fisica Nucleare, Via G. Acitelli 22, 67100 Assergi, AQ, Italy

8 LIMS, Laboratorio Nazionale del Gran Sasso-Istituto Nazionale di Fisica Nucleare, Via G. Acitelli 22, 67100 Assergi, AQ, Italy

9 Istituto Nazionale Di Fisica Nucleare-Sez. di Cagliari Monserrato, Strada Provinciale per Sestu, Km 1, 09042 Monserrato, CA, Italy

10 Centro Universitario Datazioni e Archeometria Milano, Universita degli Studi di Milano Bicocca, Piazza della Scienza 1, 20126 Milano, Italy

11 Institut Fur Geologie, Universitat Bern, Baltzerstrasse 3, 3012 Bern, Switzerland 